\section{Thomas Schierl}

\section{Werbung im Fernsehen}

Eine medienökonomische Untersuchung zur Effektivität und Effizienz werblicher TV-Kommunikation

Köln: Herbert von Helem Verlag, 2003. $239 \mathrm{~S}$.

\section{ISBN 3-931606-67-8}

Das stark anwachsende Angebot an Medienund Werbeinhalten führt zu einer Intensivierung des Wettbewerbs um die begrenzte Aufmerksamkeit von Rezipienten. Hieraus leitet sich für Werbetreibende eine Vielzahl an ökonomisch relevanten Fragestellungen hinsichtlich ihres Kommunikationsverhaltens ab, die auf ein zunehmendes Interesse in der medienökonomischen Theorie und Praxis stoßen. Thomas Schierl hat es sich zur Aufgabe gemacht, für diese beiden Zielgruppen ein Fachbuch bereitzustellen, in dessen Rahmen die Effektivität und Effizienz von werblicher TV-Kommunikation diskutiert und - hierauf aufbauend - Strategieoptionen für die Praxis vorgestellt werden. $\mathrm{Zu}$ diesem Zweck greift der Autor im Wesentlichen auf vorhandene Studien zu ausgewählten Teilaspekten zurück und interpretiert diese in Bezug auf die eigene Themenstellung. Darüber hinaus sind eigene Interviews durchgeführt worden, die ebenfalls in die Analyse werblicher TV-Kommunikation einfließen. Anzumerken ist an dieser Stelle, dass dem Fernsehen als Werbemedium eine ausgesprochen hohe Bedeutung zukommt, da es - nach Tageszeitungen-sowohl den höchsten Werbeumsatz als auch das höchste Werbewachstum aufweist.

Das Buch untergliedert sich in fünf $\mathrm{Ab}$ schnitte. Bei Abschnitt I (19 Seiten) handelt es sich um eine umfassende Einleitung, in deren Rahmen Problemstellung, Zielsetzung sowie Methodik und Aufbau der Arbeit erläutert werden. Dem Aufbau der Arbeit liegt ein selbst entwickeltes Werbewirkungsmodell zugrunde, das eine differenzierte Betrachtung des Werbeträgers Fernsehen sowie von Institutionen/Akteuren und Botschaften konstituiert. Mit Blick auf diese drei Elemente ist festzustellen, dass es sich nach Ansicht des Autors hierbei um zentrale Gestaltungs- und Einflussfelder von bzw. auf werbliche TV-Kommunikation handelt. Den Hauptteil des Buches, der die Abschnitte II, III und IV (58, 49 bzw. 102 Seiten) umfasst, bildet die Diskussion und Bewertung dieser drei Elemente im Hinblick auf die Ausgestaltung von werblicher TV-Kommunikation, die im Kern auf die Zusammenfassung und Interpretation von bereits vorliegenden Studien zurückgeht. Hierauf aufbauend werden marketingstrategische Handlungsoptionen aus Sicht von Werbetreibenden aufgezeigt und eingeordnet. Das Buch schließt mit einer Zusammenfassung und Bewertung von Kernaussagen zur Auswahl und Ausgestaltung des Werbeträgers bzw. von Institutionen/Akteuren und Botschaften (11 Seiten). Die Bewertung von Kernaussagen beinhaltet hierbei auch die Ableitung von Implikationen für Theorie und Praxis.

Das Buch ist nachvollziehbar gegliedert, gut lesbar und durch eine Vielzahl an Abbildungen und Tabellen anschaulich aufbereitet. Die inhaltlichen Ausführungen zeichnen sich durch eine intensive Einbindung aktueller Studien zu Teilaspekten sowie aussagekräftigen Zahlenmaterials aus. Aus diesem Grund leistet die Arbeit einen interessanten Beitrag in dem durch eine Vielzahl an Teilanalysen gekennzeichneten Forschungsfeld der werblichen TV-Kommunikation. Hierbei ist hervorzuheben, dass es dem Autor durch die Unterscheidung von verschiedenen Gestaltungs- und Einflussfeldern gelingt, vertiefende Einzelbetrachtungen miteinander zu verknüpfen und somit den angestrebten Werbeerfolg als Ergebnis eines multifaktoriellen Zusammenwirkens einzuordnen. Kritisch ist anzumerken, dass konkrete Anforderungen an die Optimierung von werblicher TV-Kommunikation an verschiedenen Stellen aufgezeigt, diese jedoch lediglich teilweise zu einem umfassenden Gesamtbild verdichtet und in Handlungsempfehlungen für Werbetreibende überführt werden.

Zusammenfassend ist festzuhalten, dass der Autor einen interessanten Beitrag in dem bislang in seiner Breite wenig durchdrungenen Forschungsfeld der werblichen TV-Kommunikation leistet, indem er auf Basis vorliegender Forschungsarbeiten ein neues Werbewirkungsmodell entwickelt und dessen Komponenten bzw. Gestaltungs- und Einflussfelder ausführlich erläutert. Darüber hinaus werden jeweils konkrete Anforderungen an die Optimierung von werblicher TV-Kommunikation benannt. Folglich kann das Buch sowohl als Ausgangspunkt für die Lösung praktischer Probleme der Werbegestaltung als auch für weiterführende Forschung dienen.

Thomas Hess 